See discussions, stats, and author profiles for this publication at: https://www.researchgate.net/publication/342791959

\title{
Positive Links Between Exposure to Police Violence, PTSD, and Depression Symptoms Among Yellow Vests Protesters in France
}

Article in Journal of Interpersonal Violence · July 2020

DOI: $10.1177 / 0886260520935863$

\section{CITATIONS}

3 authors:

\section{Elif Çelebi}

Marmara University

33 PUBLICATIONS 328 CITATIONS

SEE PROFILE

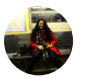

Yara Mahfud

Paris Descartes, CPSC

21 PUBLICATIONS 112 CITATIONS

SEE PROFILE

Some of the authors of this publication are also working on these related projects:
READS

197

Jaïs Adam-Troïan

Keele University

60 PUBLICATIONS 196 CITATIONS

SEE PROFILE

Project City Evaluation View project

Project Social thinking, emotions and collective behaviour in contemporary societies facing extreme situations View project 


\title{
Positive links between Exposure to Police Violence, PTSD and Depression symptoms among Yellow Vests Protesters in France.
}

\author{
Elif Çelebi ${ }^{1}$, Jais Adam-Troian² ${ }^{2}$, Yara Mahfud ${ }^{3}$
}

Corresponding Author: Jais Adam-Troian, jadam@aus.edu, American University of Sharjah, Sharjah, UAE.

Conflict of Interests: The authors have no conflict of interest to declare.

Funding: There is no funding to declare

\footnotetext{
${ }^{1}$ Department of Psychology, Istanbul Şehir University, Turkey

${ }^{2}$ Department of International Studies, American University of Sharjah, UAE

${ }^{3}$ Department of Psychology, Paris-5 University, France
} 


\title{
Positive links between Exposure to Police Violence, PTSD and Depression symptoms among Yellow Vests Protesters in France.
}

\begin{abstract}
Police violence during protests occurs worldwide with varying levels ranging from physical abuse to lethal use of force. Though prior research has investigated the health consequences of exposure to police violence (EPV), EPV's relationship with protesters' mental health has not yet been examined. Therefore, we designed a cross-sectional study which included measures of self-reported EPV, depression and post-traumatic stress symptoms. The computerized survey was disseminated among a large sample of Yellow Vests protesters in France ( $\mathrm{N}=523$; average protest attendance $=18$ ). Overall, $49 \%$ of protesters displayed severe symptoms of depression and $15.5 \%$ met the criteria for provisory PTSD diagnoses. Subsequent analyses revealed, as expected, positive associations between EPV, depression and post-traumatic stress symptoms in our sample $(.30<d<.63)$. These were robust to adjustment on several potential confounds (e.g. physical injuries, demographics, political extremism) and similar when analyzing provisory diagnoses rates $(1.65<O . R$. $<3.02)$. This study is the first to assess potentially detrimental effects of EPV on mental health during protests. Furthermore, prevalence rates for both provisory depression and PTSD diagnoses were comparable to those found among warrefugee populations. We call for further research on EPV among various protester samples and using longitudinal designs to investigate a potent risk factor for mental health issues among civilian populations.
\end{abstract}

Keywords: Police violence, Protesters, Mental health, Yellow vests, PTSD, Depression, Trauma 


\section{Introduction}

Police violence during protests occurs when law enforcement agents respond with use of force in either a proportionate or a disproportionate way. This ranges from prescribed exertion of violence on protesters to use of weapons in a way that is contrary to law enforcement's code of conduct, such as firing dispersion grenades directly at a protester. Violent protest policing occurs worldwide, with sometimes use of lethal force (e.g. 2018 Venezuelan anti-Maduro protests, 2019 anti-corruption uprising in Iraq). Though physical injuries are the most prevalent concern when it comes to violent protest policing, the issue of protesters' mental health has witnessed a recent research focus (Ni et al., 2020a; 2020b). In this paper, we assess the existence of negative associations between exposure to police violence (EPV) and mental health outcomes in the context of the Yellow Vests protests in France.

\section{Police violence during the Yellow Vests}

Starting from an initial one-time protest against raises in gas tax planned by the French government in November of 2018, the Yellow Vests movement grew into a mass social movement with weekly demonstrations up to this day. Yellow Vests protesters are mostly economically deprived citizens: $70 \%$ of them live below the median income and $24 \%$ report experiencing substantial financial difficulties (Algan, Beasley, Cohen, Foucault, \& Peron, 2019). Made of predominantly apolitical individuals (non-voters, followed by a bulk of extreme left and right wing supporters; Mahfud \& Troian, 2019), the Yellow Vest demonstrations in Paris counted approximately 300,000 protesters at their highest. The number of demonstrations and occupation of roads and roundabouts throughout metropolitan France and some overseas territory lead some to estimate an actual number of Yellow Vests at above one million. Still, no accurate number is currently available due to the fragmented and de-centralized form of this movement (Noiriel, 2019). 
A defining feature of the Yellow Vests movement has been the level of violence displayed during police-protesters encounters, which lead to more casualties and deaths than the May 1968 riots (Jetten, Mols, \& Selvanathan, 2020). The demonstrations saw a surge of police violence against protesters, with members of law enforcement using disproportionate force and weapons more powerful than during the 2005 suburb uprising (when a 'state of emergency' was declared; Chamorel, 2019). Besides physical abuse, cases of inappropriate use of French law enforcement's equipment (e.g. targeting protesters directly with distraction grenades, firing rubber pellet ammunitions to their heads) were reported, which has lead demonstrators to complain about excess use of force (see Jetten, Mols, \& Selvanathan, 2020).

The disproportionate nature of police use of force against the Yellow Vests was noticed by field observers and European institutions (with 483 cases including head wounds, comas, eye and hand losses, Paye, 2019; COE, 2019a). Public health concerns were also raised by independent observers including the United Nations' High Commission to Human Rights which wrote that 'serious injuries have been caused by a disproportionate use of so-called 'non-lethal' weapons like grenades and defensive bullets or 'flashballs" (UN, 2019; p.1). This was confirmed by subsequent research showing that the use of kinetic weapons by law enforcement (i.e. rubber ammunition launchers) had caused a surge in severe ocular injuries across French hospitals, often leading to permanent vision loss (Chauvin et al., 2019).

\section{Police violence and mental health}

Beyond the Yellow Vests context, EPV involving the use of lethal and less-lethal weapons (e.g. rubber ammunition launchers) is known to cause severe injuries including eye-losses (Lartizien et al., 2019), cranial damages and permanent disfigurements and, ultimately, death (for a detailed review of commonly used crowd-management techniques and weapons see EP, 2019). Previous research investigated the physical consequences of policing techniques used by law enforcement during protests and riots (e.g. Lavy \& Asleh, 2003), but less so their 
potential mental health consequences. Given the important human and economic cost of mental health issues (Trautmann, Rehm, \& Wittchen, 2016) and the global prevalence of police violence, clinical research investigating the psychological effects of violent protest management techniques is crucial. In fact, from a socioecological perspective (Lawson, 1992) socio-political stressors such as the degree of police use of force could be important determinants of mental health among civilian populations (Yang \& Mak, 2020).

Empirical evidence shows that traumatic events are causal factors in the development of PTSD (Pai, Suris, \& North, 2017) and are part of the stressors which increase depressive symptoms (Laugharne, Lillee, \& Janca, 2010). The $D S M-V$ defines trauma as requiring "actual or threatened death, serious injury, or sexual violence" (APA, 2013; p. 271). This definition excludes stressful but not directly life-threatening events such as psychosocial stressors (e.g., divorce). Accordingly, research shows that populations exposed to life threatening circumstances such as war veterans (Fulton et al., 2015; Xu et al., 2017) and refugees display higher levels of PTSD (Acarturk, Cetinkaya, Senay, Gulen, Aker, \& Hinton, 2018). Similarly, highly stressful life events are causally involved in $20-50 \%$ of depression cases (after a delay of approximately a month or so; see Hammen, 2005). Relatedly, the physical injuries caused by exposure to police violence (EPV) during protests might constitute traumatic events which could have consequences on protesters' mental health.

\section{The traumatic nature of police violence}

Several features of EPV may explain how they could constitute traumatic experiences for protesters and affect their mental health. First, EPV can take the form of physical brutality and abuse, which is already included as a PTSD trauma criterion in the DSM-V. During the Yellow Vest protests for instance, an estimated number of 2 people died and 4190 were injured due to direct or indirect actions from law enforcement (including physical brutality and abuse, Mahfud $\&$ Troian, 2019; Jetten et al., 2020). Another traumatic aspect of law enforcement's display of 
force in protests is the kind of weapons they use for crowd-management. For example, as of February of 2019, French law enforcement had used more than 12,000 rubber pellet rounds (causing 190 severe injuries, Chauvin et al., 2019), 1400 tear gas and 4942 sting grenades in Yellow Vests protests. These latter grenades are responsible for cases of hand loss $(\mathrm{N}=5$; for a detailed review see COE, 2019b). The use of military equipment, the frequency of detonations, fires and violent clashes between protesters and law enforcement officers all indicate that some features of protests may be comparable to those found in armed conflicts, thus likely to constitute traumatic experiences. Recent investigations of the Hong Kong antiextradition bill protests highlighted a detrimental effect of protest attendance on mental health outcomes (PTSD and depression symptoms; see Ni et al., 2017, 2020a; also see Hou, \& Hall, 2019). In these samples, prevalence of PTSD and depression was comparable to that observed among war-veterans (respectively $11.2 \%$ and $12.8 \%$ ), a relevant comparison group given the shared features of violent protests and war contexts (as well as the traumatic role of interpreting these as 'malevolent' environments, see Lee, Aldwin, \& Kang, 2020). Although these studies discussed the potentially traumatic nature of protests by focusing on the stressful character of protest contexts (looting, crowd movements, unusual noise, fires; Ni et al., 2020a), the effect of direct exposure to police violence among protesters had yet to be examined.

\section{The present research}

Studies on trauma and mental health focus mostly on crime and war-related contexts (e.g. Bhi et al., 2003) while research on EPV's health consequences was mainly limited to policeminority encounters within community settings (mostly in the US; Cooper, Moore, Gruskin, \& Krieger, 2004). Because EPV may be a potential trauma with mental health consequences for protesters (i.e. a risk factor for mental health issues), we decided to conduct a pioneer casecontrol study among a protester sample. The aim of this investigation was to explore whether 
EPV - in the form of physical abuse or less-lethal weapons - during protests could be associated with negative mental health outcomes, namely depression and PTSD.

\section{Methods}

\subsection{Ethical statement}

The study was conducted in accordance with the 1964 Helsinki declaration and its later amendments, the French legislation on research involving human participants and the 2016 APA Ethical Principles of Psychologists and Code of Conduct. Ethic approval was obtained from [ANONYMIZED INSTITUTION] ethics board (n²9-2019).

\subsection{Participants and procedure}

Target population. We aimed to collect data from a representative sample of individuals involved in the Yellow Vests protests, albeit limited to those individuals able to access online information and to participate in online surveys (see 'Procedure' subsection below).

Analytical Strategy. In order to make reliable inferences, we needed to demonstrate that results converged across different types of analyses. In a first set of analyses, correlations between our measures of EPV and of psychological disorders would be conducted. We would then create EPV categories by crossing EPV measures $(n=4$; no exposure, exposure to police brutality only, to less-lethal weapons only and to both). Subsequent tests would be performed using the categories derived from EPV measures as an independent variable and continuous mental health symptoms as dependent variables (ANOVA). Finally, these tests would be replicated using the same categorical independent variable and provisory diagnoses - based on measures of psychological disorders - as the dependent variables (Chi-square). Whenever possible, supplementary tests would be performed again with a number of relevant covariates (i.e. demographics, exposure to non police-related traumas, political ideology) to check results' robustness and eliminate concerns regarding potential confounds (i.e. sensitivity analyses). 
Power analyses. The design was cross-sectional. To ensure stable parameter estimates, at least 250 respondents were needed (see Schönbrodt, \& Perugini, 2013). We set our smallest effect size of interest based on up-to date benchmarks for psychological research (Funder, \& Ozer, 2019) to $r=.20$, indicating medium effects with practical use in the short and long run (appropriate for assessing mental health risk factors in the context of single and repeated protest attendance). Power analyses were conducted using Gpower (Faul, Erdfelder, Buchner, \& Lang, 2009), for a power set at $80 \%$, an alpha level at 5\% using two-tailed tests. We found that, with an expected $r=.20,255$ participants were needed for correlation analyses. At least 95 participants per EPV category would be needed to conduct post-hoc t-tests $(d=.41)$, leading to a required sample size up to almost $N=400$. Therefore, we aimed for a sample size of $N=500$ to ensure adequate power across a wide range of statistical tests.

Procedure. Links to an online survey were disseminated on Yellow Vests social media pages by the investigators. Leaders of the movement and administrators of webpages and forums dedicated to the Yellow Vests were also asked to voluntary disseminate survey links. Data was collected during the summer of 2019. Investigators frequently monitored the sample size and stopped collection when the expected sample size of at least 503 Yellow Vest participants was reached.

Sample. Respondents' self-reported identification as a Yellow Vest member was assessed by a categorical (binary) item, while number of protests attended was measured continuously. Out of 598 respondents, we retained answers from $523(87.45 \%)$ respondents who selfidentified as Yellow Vests $\left(48.7 \%\right.$ male; $\left.M_{\mathrm{age}}=44.0, S D=12.6\right)$. Respondents attended 18.0 protests on average (Ranging from ' 0 ' to ' 79 '; $S D=12.7$ ).

[INSERT TABLE 1 HERE]

\subsection{Materials}


The survey contained two parts, one epidemiological and one psychosocial. The psychosocial part of the survey included measures related to protest behavior (e.g. intentions to self-sacrifice, to protest in the future...) designed to address other research questions. The epidemiological part of the survey examined in this study contained the following measures:

Exposure to Police Violence Traumas. EPV was measured with two binary items regarding physical violence. In order to avoid negativity biases in reporting due to subjective labels (e.g. asking participants if they had been exposed to 'police brutality'), we worded the two items as factually as possible. Accordingly, participants were asked if - during their participation in YV protests - they had 'ever been exposed to physical violence coming from law enforcement in the form of being pushed, beaten and/or beaten with a baton' and if they had 'ever been exposed to a rubber pellet ammunition shot coming from law enforcement' (see Kubany et al., 2000). Among all the protests they attended, 58.2\% $(n=302)$ reported to have been exposed to Police Brutality at least once and $25.3 \%(n=131)$ had been hit by a Rubber Ammunition.

We then classified participants according to their levels of exposure to both Police Brutality and Rubber Ammunitions. Among them, 37.5\% $(n=193)$ declared no exposure, $37.3 \%(\mathrm{n}=$ 192) reported exposure to Police Brutality only, 4.5\% $(n=23)$ exposure to Rubber Ammunition only and $20.8 \%$ declared having been exposed to both forms of police violence. Cases of exposure to Rubber Ammunition only were few $(n=23)$ and the two types of exposure substantially overlapped $\chi^{2}(1)=42.0, p<.001$. Thus, participants exposed to Rubber Ammunitions were also more likely to suffer from Police Brutality, which prevented us from running analyses allowing to reliably disentangle these two factors.

Because of this unexpected empirical constraint, we decided to base our categories on the number of exposures to police-related traumas (ranging from 0 to 2; as opposed to categories based on the type of exposure to police trauma). In total, $37.5 \%$ of respondents $(n=193)$ had not been exposed to police violence, $41.7 \%(n=215)$ reported at least one exposure and $20.8 \%$ 
$(n=107)$ reported both. This method of classification ensured optimal power for our tests including independent t-tests - which required a minimum of 95 subjects per comparison group (see the 'Power analyses' section).

Exposure to Protest-related Traumas. In addition, we measured participants' exposure to other protest-related potential traumas, namely exposure to Teargas $(85.8 \%, n=448)$, having suffered from Minor Injuries (as indicated by a temporary work incapacity; 6.9\%, $n=36$ ) or Severe Injuries (hospitalization; 3.8\%, $n=20$ ), as well as participants' experience of being brought into Custody $(13.8 \%, n=72)$. Measuring traumas related to the protest context and not specifically to EPV would allow to assess various risk factors and take into account potential confounding effects.

Depression. Depressive symptoms were measured with the HSCL-25, which is a widely used tool to assess intensity of anxiety and depression symptoms (25 items, 4-point Likert, $\alpha=$ .94, $M=1.79, S D=.64$; Fröjdh, Håkansson, \& Karlsson, 2004). The French version has a recommended cutoff score of 1.75 , which yields a Sensitivity $=59.1 \%$ and a Specificity $=$ 91.4\% when diagnosing severe symptoms of depression (Nabbe et al., 2019). Using this cutoff score, $49 \%(n=256)$ of our sample presented severe symptoms of depression.

PTSD. PTSD symptoms were assessed with the PCL-5. This standard scale contains 20 items corresponding to the 20 criteria for PTSD from the DSM-5 organized in four symptom clusters (5-point Likert, $\alpha=.95, M=1.09, S D=.95$; Weathers, Litz, Keane, Palmieri, Marx, \& Schnurr, 2013). The French version of this scale displayed a Sensitivity $=83 \%$, and a Specificity $=95 \%$ for PTSD (Ashbaugh, Houle-Johnson, Herbert, El-Hage, \& Brunet, 2016). To compute severe PTSD symptoms (i.e. provisory PTSD 'diagnosis'), we proceeded in line with the DSM5 criteria. To meet the criteria for a provisory PTSD diagnosis, participants needed 1) to have been exposed to least one potentially traumatic event 2) at least one month prior to assessment and 3) to display severe symptoms of PTSD (as indicated by a sum score above the scale's 
cutoff score; APA, 2013; also see USDVA, 2019). Using the symptom severity cutoff of score of 31 provided by Ashbaugh et al., (2016) for their French version of the PCL-5 this procedure yielded a tentative PTSD rate of $15.5 \%(n=81)$ in our sample.

Confounding factors. Due to the cross-sectional character of our investigation, we decided to run sensitivity analyses for each of our tests adjusting on a host of potential confounds. In addition to other demographics, respondents were asked to indicate their yearly household income (5-levels from 'less than 30,000€' to 'more than 120,000€'; Median =1, <30,000€'). Besides adjustment on sample characteristics (age, gender, previous number of protests attended and income), we also decided to leverage a measure of political ideology from the socio-political part of the survey. It contained a single item (Likert-7 points; from 1 'far-left' to 7 'far-right', $M=3.07, S D=1.49$ ) from which we also computed a measure of political extremism, assessing individual score's distance from the scale's midpoint (' 4 ' recoded ' $O$ '; with a scale maximum $=3, M=1.32, S D=1.15$ ). Ideology would adjust for potential selfreport biases due to participants' political orientation (e.g. strong anti-government attitudes), while political extremism is linked with increased intentions to engage in illegal and violent forms of collective action (including assaulting law enforcement; Mahfud \& Troian, 2019). Controlling for extremism would therefore allow to - partially - rule out spurious results from extreme individuals who engage more often in illegal actions and are thus more exposed to other traumas prior to EPV (criminal violence, prison...).

\section{Results}

\subsection{Correlation analyses}

Point-biserial correlations were computed to investigate the links between symptom levels (HSCL and PCL scores) and exposure to both EPV and protest-related traumas. As can be seen in table 2, most protest-related traumas (except for custody) were significantly associated with both HSCL and PCL scores. As expected, exposure to Police Brutality was linked with both 
both PCL, $r(510)=.33, p<.001$ and HSCL scores, $r(512)=.29, p<.001$. Exposure to Rubber Ammunitions was also positively associated with PCL, $r(509)=.14, p<.001$ and HSCL scores, $r(511)=.16, p<.001$.

\section{[INSERT TABLE 2 HERE]}

To assess the robustness of these links, partial point-biserial correlations were computed between both EPV measures, PCL and HSCL scores, controlling for protest-related traumas, number of protest previously attended, age, gender, income, political extremism and ideology. Exposure to Police Brutality was still linked with PCL, $r(492)=.23, p<.001$ and HSCL scores, $r(492)=.20, p<.001$. However the link between exposure to Rubber Ammunitions and HSCL scores was weaker, $r(492)=.09, p=.054$ and negligible regarding PCL scores $r(492)=.05$, $p=.24$. Because of the limited information brought by simple correlation analyses, we turned to comparisons between categories from our aggregated measure of EPV number (see '2.3. Materials').

\subsection{Exposure group comparisons}

HSCL and PCL scores. A one-way ANOVA indicated the presence of a meaningful association between EPV levels and HSCL scores, $F(2,505)=23.50, p<.001, \eta^{2}=.09$. This effect was robust to inclusion of other protest-related traumas, number of protest previously attended, age, gender, income, political extremism and ideology as covariates (ANCOVA), $F(2$, 494) $=8.59, p<.001, \eta^{2}=.03$. Thus, we proceeded to pairwise comparisons using Holm correction for type I error control (Aickin \& Gensler, 1996). As can be seen in figure 1, HSCL scores were substantially higher among individuals reporting one EPV trauma, $M=1.86(S D=$ $.59)$, compared to non-exposed individuals, $M=1.57(S D=.57), t(505)=4.85, p<.001, d=$ .48. Similarly, individuals reporting two EPV trauma $M=2.04(S D=.70)$, had higher HSCL scores than those reporting only one, $t(505)=2.54, p=.011, d=.30$. 
Similar tests revealed an association between EPV levels and PCL, $F(2,503)=29.70, p<$ $.001, \eta^{2}=.11$, which were also robust to adjustment on previously used covariates, $F(2,492)=$ 11.03, $p<.001, \eta^{2}=.04$. Pairwise comparisons indicated that PCL scores too were higher among individuals reporting one EPV trauma $M=1.26(S D=.90)$, compared to non-exposed individuals, $M=.69(S D=.82), t(503)=6.35, p<.001, d=.63$. However, being exposed to two EPV traumas only marginally increased PCL scores, $M=1.42(S D=1.02), t(503)=1.51$, $p=.13, d=.18$ compared to one EPV (see figure 1 ).

\section{[INSERT FIGURE 1 HERE]}

Depression and PTSD rates. Because there may exist differences depending on the way data is coded and analysed, we wanted to provide another assessment of the link between EPV and mental health outcomes. One way to do so was to run similar tests as previously but using categorical tentative diagnoses and see if results converged. We thus performed chi-square tests to assess the link between EPV and both Depression and PTSD rates.

EPV was linked with Depression rates, $\chi^{2}(2)=29, p<.001$. Mirroring the results obtained using symptom severity scores, individuals exposed to one EPV trauma had higher Depression rates, $\%_{\mathrm{DEP}}=53.5$, than non-exposed individuals, $\%_{\mathrm{DEP}}=34.7, O . R .=2.16, \chi^{2}(1)=14.5, p<$ 0.001, but lower than those exposed to two EPV traumas, \% DEP $=65.4, O . R .=1.65, \chi^{2}(1)=$ 4.16, $p=0.041$ (see figure 2).

\section{[INSERT FIGURE 2 HERE]}

To assess the associations between EPV and PSTD tentative rates, we had to proceed differently. In fact, PTSD rates can only be computed among individuals exposed to at least one trauma of some sort, so we restricted the analysis to these cases only ( $86 \%$ of our sample; $n=451)$. Among them, we then compared individuals who reported exposure to protest-related trauma (teargas, light injury, severe injury and custody, $28.8 \% ; n=130$ ) to those who reported 
EPV traumas (rubber pellets and police brutality, 71.2\%; $\mathrm{n}=321$ ). We found that individuals exposed to EPV traumas had substantially higher PTSD rates, \% $\mathrm{PTSD}=21.8$, than those exposed to other types of protest-related traumas, $\%$ PTSD $=8.5, O . R .=3.02, \chi^{2}(1)=11.2, p<.001$ (see figure 3).

\section{[INSERT FIGURE 3 HERE]}

\section{Discussion}

As expected from previous research investigating traumatic contexts and mental health issues, our study highlighted strong positive associations between EPV and prevalence of psychological disorders among a sample of Yellow vests protesters. These results constitute first evidence that self-reported levels of EPV are associated with increased PTSD and Depression symptoms among protest participants. Moreover, our findings were robust to adjustments on various potential confounds and converged across different analyses using both continuous symptoms and categorical provisory diagnoses rates as outcomes. Importantly, we were able to replicate findings from the trauma literature in a different context (protests).

As an illustration, observed depression rate in our sample (49\%) was slightly more than twice the prevalence rate among the French population (around 20\%; see Gigonzac, Delézire, \& Khireddine-Medouni, 2018) and close to the lower end estimates of depression symptoms among war refugees (ranging from $40 \%$ to more than $80 \%$; e.g. Bogic, Njoku, \& Priebe, 2015; Vonnahme, Lankau, Ao, Shetty, \& Cardozo, 2015). Similarly, the rate of PTSD provisory diagnoses we estimated (15.5\%) was comparable to those found among Hong Kong protesters using the same measure (12.8\%; Ni et al., 2020). It was also well within the range of what is commonly found in other samples exposed to traumatic contexts involving armed violence, injuries and fatalities (e.g. 9-36\% among war refugees, Turrini, Purgato, Ballette, Nosè, Ostuzzi, \& Barbui, 2017; 9-30.9\% among US war veterans, Dohrenwend, Turner, Turse, Adams, Koenen, \& Marshall, 2006). 
Another crucial finding is that depression symptoms displayed a proportional increase according to EPV levels. Since proportionality (or so-called 'dose-response') is an important criterion for establishing causal associations in epidemiology (Hill, 1965; Rothman \& Sander, 2005), our results are encouraging with regards to the presence of potential causal links. This proportionality was less clear for post-traumatic stress symptoms $(\mathrm{p}=.13$, two-tails or .065 , one-tail). However, this does not necessarily mean that causal relationships regarding EPV and PTSD are unclear. It is possible that one police-related traumatic experience is necessary and sufficient to trigger subsequent PTSD (non-linear effect) but our study design does not allow to make any further claims regarding this issue.

In a similar way, though EPV was designed to tap into two very different types of exposures (rubber ammunitions and abuse/brutality), we could not really make use of this distinction. Individuals exposed to rubber ammunitions were also much more likely to also report physical abuse and brutality, which did not allow us to disentangle the specific effects of these two types of police violence. This may be because individuals hit by rubber ammunitions are more likely to be involved in violent contacts with law enforcement, or more likely to be violently treated/arrested subsequently. Though experience of injuries, custody and political extremism were used as covariates and ruled out as confounds of the links between EPV and mental health outcomes, we were still left with an important empirical issue. Future qualitative or archival research could help to highlight how various type of EPV ecologically relate to each other and ultimately lead to the design of more accurate measurements. Relatedly, individual perceptions of trauma ( $v s$. facts) do matter in the formation of mental health issues (e.g. Danese \& Widom, 2020). Choosing not to use subjective labels to assess EPV may have contributed to underestimate the size of the association between EPV and mental health symptoms (Goodman, Corcoran, Turner, Yuan, \& Green, 1998).

\subsection{Limitations}


Despite these encouraging first results this study contained important caveats that constrain how results can be generalized. First and foremost, because the design was cross-sectional, causality should be discussed with caution. Though there are strong theoretical and empirical priors to support a causal direction from EPV to mental health outcomes, and although we used several covariates, alternative hypotheses may still be considered. For instance, some covariates measured exposure to other protest-related traumas, but not participant's prior experience of traumatic events. Mental health issues might sometimes be associated with violent extremism (Gøtzsche-Astrup, \& Lindekilde, 2019) and prior experience of traumatic events could generate more violent engagement with the police, hence leading to our observation of a link between EPV and degraded mental health. Though we have reasons to believe this is very unlikely, future studies using longitudinal designs should be conducted to rule out this possibility.

Second, even if in the range of what is found in the literature on trauma contexts and mental health, depression symptoms were high in our sample. This is probably due to a combination of several factors. First, our sample was mostly made of low-income participants, which are already more at risk for mental health issues (Marmot \& Wilkinson, 2003) and would have been hit harder than the rest of the population by Macron's gas tax increase (Jetten et al., 2020). Likewise, protesters in our sample attended 18 demonstrations on average and chronic stress is a risk factor for increased onset, maintenance, and recurrence of major depression (Monroe \& Harkness, 2005).

The online nature of our survey may have also affected our point estimates of psychological disorders, although research shows that risk factor estimates (the link between EPV and outcomes) from online and offline studies are similar (see Heiervang, \& Goodman, 2011). Other response biases might have been induced because some Yellow Vests - due to their political status - are afraid of being 'monitored' by the government. It is thus likely that more radical protesters might not have taken the survey. Among those who did respond, some may have 
guessed the research hypothesis and answered in a socially desirable manner, potentially generating a stronger link between EPV and mental health outcomes. Although this possibility cannot be completely excluded, adjustment on political ideology/extremism did not affect our results, rendering less likely that political motivations distorted participants' answers. In addition to being members of the Yellow Vests, some participants may belong to other vulnerable populations (e.g. war veterans) which could further bias our estimates. Last but least, we used cutoff scores for the HSCL that were determined on non-protester populations.

Therefore, subsequent field investigations should be carried out using rigorous diagnostic interviews with trained assessors to estimate true depression (and potentially PTSD) prevalence rates among protesters. Observational data could also be collected during protests to classify frequent situations and police-protester behaviours that may constitute traumatic experiences specific to protest contexts. Issues regarding the diversity of protesters investigated should be taken into account: prevalence rates should be estimated in a wide range of different cultural contexts (where protest policing and social issues may vary), as well as for different groups within protester samples (e.g. stigmatized minorities, which may also have prior experience of EPV and unfair treatment by law enforcement). This could be done through comparative survey studies involving large international samples, preferably among protesters from populations representing the whole cultural distance spectrum (see Muthukrishna et al., 2018) and would help determining more specific cut-off scores for future research. Additionally, the moderating role of income, education and SES on the link between protest experience (including EPV) and mental health outcomes could be investigated using a mix of crowd surveys among protesters and experimental paradigms manipulating both income allocation and exposure to various protest-related stressors (Fisher et al., 2019).

Nevertheless, even if prevalence rates for Depression and PTSD provided in this study are skewed, we consider it as a first successful test of the hypothesized associations between EPV 
and prevalence of psychological disorders. Moreover, this test was strong because we were still able to detect some effect of EPV among a sample where one might expect ceiling effects (i.e. low income protesters).

\subsection{Practical Implications}

Within these inferential boundaries, this study showed that EPV could a be an important risk factor for clinically significant mental health issues. Therefore, while waiting for further assessments of EPV as a risk factor for mental health disorders, preventive actions to contain police violence should be taken whenever possible. Promoting crowd-management techniques such as 'de-escalation' (Engel, McManus, \& Herold, 2020) and conducting moratoriums on the use of less lethal weapons such as rubber ammunition launchers could be found useful. Adapting law enforcement trainings to make them engage in more respectful, neutral and transparent protest policing (i.e. procedural justice trainings), or in positive non-enforcement interactions with protesters could also significantly reduce both EPV and protester violence (Peyton, Sierra-Arévalo, \& Rand, 2019; Wood, Tyler, \& Papachristos, 2020). Interventional studies could also assess the effectiveness of non-retaliation mediation techniques from gang violence prevention research translated to police-protesters relations (see Brantingham et al., 2018).

More research would be also needed to address the current gap in the literature regarding healthcare access for protesters. Access to mental health care is indeed dependent on countryspecific factors such as the type health care system in place (e.g. universal health care in France) but also population prevalence of mental health practitioners (which may be problematic in some countries, see Bruckner et al., 2011). Our study points at the urgency of investigating mental healthcare access for protesters, especially given converging findings that protest contexts can have a detrimental effect (Ni et al., 2020b). Trauma emergency interventions have displayed limited efficacy (mostly for depressive symptoms; Laugharne et al., 2010). Therefore, 
further research is needed to assess the feasibility of implementing novel group interventions in post-protest contexts (e.g. Haslam, Cruwys, Haslam, Dingle, \& Chang, 2016; Haslam et al., 2019) and effectiveness of psychological training for so-called 'street-medics' in providing emergency psychological care to traumatized protesters.

\subsection{Conclusion}

Our study highlights the pressing need for research investigating the effects of crowdmanagement techniques on citizens' mental health in order to improve protest policing. We hope this issue be the focus of future large-scale observational studies, epidemiological assessments and intervention research. Due to its large impact, crowd management could be an important target area for improving global mental health (Collins et al., 2011), and we believe the present study provides evidence for policy and practice changes that may ultimately benefit both protesters and law enforcement.

\section{References}

Acarturk, C., Cetinkaya, M., Senay, I., Gulen, B., Aker, T., \& Hinton, D. (2018). Prevalence and predictors of posttraumatic stress and depression symptoms among Syrian refugees in a refugee camp. The Journal of nervous and mental disease, 206(1), 40-45.

Aickin, M., \& Gensler, H. (1996). Adjusting for multiple testing when reporting research results: the Bonferroni vs Holm methods. American journal of public health, 86(5), 726728.

Algan, Y., Beasley, E., Cohen, D., Foucault, M., \& Peron, M. (2019). Qui sont les Gilets jaunes et leurs soutiens? [Who are the Yellow Vests and their supporters?] (CEVIPOF Working Paper No. 2019-03). Accessed at 
http://www.sciencespo.fr/cevipof/sites/sciencespo.fr.cevipof/files/-Qui-sontles-Giletsjaunes-et-leurs-soutiens-1.pdf

American Psychiatric Association (2013). Diagnostic and Statistical Manual of Mental Disorders. 5th ed. American Psychiatric Association: Arlington, VA, USA.

Ashbaugh, A. R., Houle-Johnson, S., Herbert, C., El-Hage, W., \& Brunet, A. (2016). Psychometric validation of the English and French versions of the posttraumatic stress disorder checklist for DSM-5 (PCL-5). PloS one, 11(10), e0161645.

Beckham, J. C. (2015). The prevalence of posttraumatic stress disorder in Operation Enduring Freedom/Operation Iraqi Freedom (OEF/OIF) veterans: A meta-analysis. Journal of anxiety disorders, 31, 98-107.

Bhui, K., Abdi, A., Abdi, M., Pereira, S., Dualeh, M., Robertson, D., ... \& Ismail, H. (2003). Traumatic events, migration characteristics and psychiatric symptoms among Somali refugees. Social psychiatry and psychiatric epidemiology, 38(1), 35-43.

Bogic, M., Njoku, A., \& Priebe, S. (2015). Long-term mental health of war-refugees: a systematic literature review. BMC international health and human rights, 15(1), 29.

Brantingham, P. J., Yuan, B., Sundback, N., Schoenberg, F. P., Bertozzi, A. L., Gordon, J., ... \& Malinowski, S. (2018). Does violence interruption work?. Proceedings of the National Academy of Science of the United States of America, 1-6.

Bruckner, T. A., Scheffler, R. M., Shen, G., Yoon, J., Chisholm, D., Morris, J., ... \& Saxena, S. (2011). The mental health workforce gap in low-and middle-income countries: a needs-based approach. Bulletin of the World Health Organization, 89, 184-194.

Chamorel, P. (2019). Macron Versus the Yellow Vests. Journal of Democracy, 30(4), 48-62. 
Chauvin, A., Bourges, J. L., Korobelnik, J. F., Paques, M., Lebranchu, P., Villeroy, F., ... \& Mouriaux, F. (2019). Ocular injuries caused by less-lethal weapons in France. The Lancet, 394(10209), 1616-1617.

Collins, P. Y., Patel, V., Joestl, S. S., March, D., Insel, T. R., Daar, A. S., ... \& Glass, R. I. (2011). Grand challenges in global mental health. Nature, 475(7354), 27.

Cooper, H., Moore, L., Gruskin, S., \& Krieger, N. (2004). Characterizing perceived police violence: implications for public health. American Journal of Public Health, 94(7), 1109-1118.

Council of Europe (2019, february). Memorandum on maintaining public order and freedom of assembly in the context of the "yellow vest" movement in France. Accessed at https://rm.coe.int/commdh-2019-8-memorandum-france-en/1680932f57

Danese, A., \& Widom, C. S. (2020). Objective and subjective experiences of child maltreatment and their relationships with psychopathology. Nature Human Behaviour, $1-8$.

Dohrenwend, B. P., Turner, J. B., Turse, N. A., Adams, B. G., Koenen, K. C., \& Marshall, R. (2006). The psychological risks of Vietnam for US veterans: a revisit with new data and methods. Science, 313(5789), 979-982.

Engel, R. S., McManus, H. D., \& Herold, T. D. (2020). Does De-escalation Training Work? Criminology and Public Policy, 1.

European Parliament (2019a). Motion for a resolution $N^{\circ}$ B8-0104/2019.

https://www.europarl.europa.eu/doceo/document/B-8-2019-0104_EN.html. Published February, 2019, accessed May, 2020. 
European Parliament (2019b). Crowd Control Technologies - An Assessment Of Crowd

Control Technology Options For The European Union. http://www.europarl.europa.eu/RegData/etudes/etudes/stoa/2000/168394/DG-4STOA_ET(2000)168394_EN(PAR02).pdf. Published June, 2000, Accessed September 6, 2019.

Faul, F., Erdfelder, E., Buchner, A., \& Lang, A. G. (2009). Statistical power analyses using G* Power 3.1: Tests for correlation and regression analyses. Behavior research methods, 41(4), 1149-1160.

Fisher, D. R., Andrews, K. T., Caren, N., Chenoweth, E., Heaney, M. T., Leung, T., ... \& Pressman, J. (2019). The science of contemporary street protest: New efforts in the United States. Science advances, 5(10), eaaw5461.

Fond, G., Lancon, C., Auquier, P., \& Boyer, L. (2019). Prevalence of major depression in France in the general population and in specific populations from 2000 to 2018: A systematic review of the literature. Presse medicale (Paris, France: 1983), 48(4), 365375.

Fröjdh, K., Håkansson, A., \& Karlsson, I. (2004). The Hopkins Symptom Checklist-25 is a sensitive case-finder of clinically important depressive states in elderly people in primary care. International Journal of Geriatric Psychiatry, 19(4), 386-390.

Fulton, J. J., Calhoun, P. S., Wagner, H. R., Schry, A. R., Hair, L. P., Feeling, N., ... \& Beckham, J. C. (2015). The prevalence of posttraumatic stress disorder in Operation Enduring Freedom/Operation Iraqi Freedom (OEF/OIF) veterans: A metaanalysis. Journal of anxiety disorders, 31, 98-107. 
Funder, D. C., \& Ozer, D. J. (2019). Evaluating effect size in psychological research: Sense and nonsense. Advances in Methods and Practices in Psychological Science, 2(2), 156-168.

Gigonzac, V., Delézire, P., Khireddine-Medouni, I. (2018). La dépression dans la population active occupée en France en 2017. Baromètre santé 2017. [Depression among the French active population in 2017. 2017 Health Barometer]. Bulletin Epidemiologique Hebdomadaire, 32(33), 645-52.

Global Burden of Disease Collaborative Network (2018). Global Burden of Disease Study 2017 Results. United States: Institute for Health Metrics and Evaluation (IHME): Seattle.

Goodman, L. A., Corcoran, C., Turner, K., Yuan, N., \& Green, B. L. (1998). Assessing traumatic event exposure: General issues and preliminary findings for the Stressful Life Events Screening Questionnaire. Journal of Traumatic Stress: Official Publication of The International Society for Traumatic Stress Studies, 11(3), 521542.

Gøtzsche-Astrup, O., \& Lindekilde, L. (2019). Either or? Reconciling findings on mental health and extremism using a dimensional rather than categorical paradigm. Journal of forensic sciences, 64(4), 982-988.

Hammen, C. (2005). Stress and depression. Annu. Rev. Clin. Psychol., 1, 293-319.

Haslam, C., Cruwys, T., Haslam, S. A., Dingle, G., \& Chang, M. X. L. (2016). Groups 4 
Health: Evidence that a social-identity intervention that builds and strengthens social group membership improves mental health. Journal of affective disorders, 194, 188195.

Haslam, C., Cruwys, T., Chang, M. X. L., Bentley, S. V., Haslam, S. A., Dingle, G. A., \& Jetten, J. (2019). GROUPS 4 HEALTH reduces loneliness and social anxiety in adults with psychological distress: Findings from a randomized controlled trial. Journal of consulting and clinical psychology, 87(9), 787.

Heiervang, E., \& Goodman, R. (2011). Advantages and limitations of web-based surveys: evidence from a child mental health survey. Social psychiatry and psychiatric epidemiology, 46(1), 69-76.

Hill, A. B. (1965). The Environment and Disease: Association or Causation? President's Address. Proceedings of the Royal Society of Medicine, 58, 295-300.

Hou, W. K., \& Hall, B. J. (2019). The mental health impact of the pro-democracy movement in Hong Kong. The Lancet Psychiatry, 6(12), 982.

Jetten, J., Mols, F., \& Selvanathan, H. P. (2020). How Economic Inequality Fuels the Rise and Persistence of the Yellow Vest Movement. International Review of Social Psychology, 33(1).

Kubany, E. S., Leisen, M. B., Kaplan, A. S., Watson, S. B., Haynes, S. N., Owens, J. A., \& Burns, K. (2000). Development and preliminary validation of a brief broad-spectrum measure of trauma exposure: the Traumatic Life Events Questionnaire. Psychological assessment, 12(2), 210.

Lartizien, R., Schouman, T., Raux, M., Debelmas, A., Lanciaux-Lemoine, S., Chauvin, A., ... 
\& Bertolus, C. (2019). Yellow vests protests: facial injuries from rubber bullets. The Lancet, 394(10197), 469-470.

Laugharne, J., Lillee, A., \& Janca, A. (2010). Role of psychological trauma in the cause and treatment of anxiety and depressive disorders. Current Opinion in Psychiatry, 23(1), 2529.

Lawson, H. A. (1992). Toward a socioecological conception of health. Quest, 44(1), 105-121.

Lavy, T., \& Asleh, S. A. (2003). Ocular rubber bullet injuries. Eye, 17(7), 821.

Lee, H., Aldwin, C. M., \& Kang, S. (2020). Do different types of war stressors have independent relations with mental health? Findings from the Korean Vietnam Veterans Study. Psychological Trauma: Theory, Research, Practice, and Policy. Advance online publication.

Mahfud, Y., \& Adam-Troian, J. (2019). “Macron demission!”: Loss of significance generates violent extremism for the Yellow Vests through feelings of anomia. Group Processes \& Intergroup Relations. Advanced online publication

Marmot, M., \& Wilkinson, R. G. (Eds.). (2003). Social determinants of health: the solid facts. Copenhagen: World Health Organization.

Monroe, S. M., \& Harkness, K. L. (2005). Life stress, the" kindling" hypothesis, and the recurrence of depression: considerations from a life stress perspective. Psychological review, 112(2), 417.

Muthukrishna, M., Bell, A. V., Henrich, J., Curtin, C. M., Gedranovich, A., McInerney, J., \& 
Thue, B. (2018). Beyond WEIRD psychology: measuring and mapping scales of cultural and psychological distance. Available at SSRN 3259613.

Nabbe, P., Le Reste, J. Y., Guillou-Landreat, M., Gatineau, F., Le Floch, B., Montier, T., ... \& Van Royen, P. (2019). The French version of the HSCL-25 has now been validated for use in primary care. PloS one, 14(4), e0214804.

Ni, M. Y., Li, T. K., Pang, H., Chan, B. H., Kawachi, I., Viswanath, K., ... \& Leung, G. M. (2017). Longitudinal patterns and predictors of depression trajectories related to the 2014 occupy central/umbrella movement in Hong Kong. American journal of public health, 107(4), 593-600.

Ni, M. Y., Yao, X. I., Leung, K. S., Yau, C., Leung, C. M., Lun, P., ... \& Leung, G. M. (2020a). Depression and post-traumatic stress during major social unrest in Hong Kong: a 10-year prospective cohort study. The Lancet.

Ni, M. Y., Kim, Y., McDowell, I., Wong, S., Qiu, H., Wong, I. O., .. \& Leung, G. M. (2020b). Mental health during and after protests, riots and revolutions: A systematic review. Australian \& New Zealand Journal of Psychiatry, 54(3), 232-243.

Noiriel, G. (2019). Les Gilets jaunes à la lumière de l'histoire [A Historical Highlight of The Yellow Vests]. Paris: Editions de l'aube.

Pai, A., Suris, A. M., \& North, C. S. (2017). Posttraumatic stress disorder in the DSM-5: controversy, change, and conceptual considerations. Behavioral Sciences, 7(1), 7.

Paye, J.-C. (2019). The yellow vests in France. Archive.monthlyreview.org.

Peyton, K., Sierra-Arévalo, M., \& Rand, D. G. (2019). A field experiment on community 
policing and police legitimacy. Proceedings of the National Academy of Sciences, 116(40), 19894-19898.

Rothman, K.J., \& Sander G. (2005). Causation and Causal Inference in Epidemiology. American Journal of Public Health, 95 (1), 144-150.

Schönbrodt, F. D., \& Perugini, M. (2013). At what sample size do correlations stabilize? Journal of Research in Personality, 47(5), 609-612.

Trautmann, S., Rehm, J., \& Wittchen, H. U. (2016). The economic costs of mental disorders. EMBO reports, 17(9), 1245-1249.

Turrini, G., Purgato, M., Ballette, F., Nosè, M., Ostuzzi, G., \& Barbui, C. (2017). Common mental disorders in asylum seekers and refugees: umbrella review of prevalence and intervention studies. International Journal of Mental Health Systems, 11(1), 51.

United Nations (2019). Human Rights Office of the High Commissioner Website. https://www.ohchr.org/EN/NewsEvents/Pages/DisplayNews.aspx?NewsID=24166\&L angID=E\&fbclid=IwAR3DTxysmOwEDjJHPU4d0RiIfg8zWmhtHV7NPIT9vx2DFdLCd3wRNTaE68. Accessed September 6, 2019.

U.S. Department of Veteran Affairs (2019). PTSD Checklist for DSM-5 (PCL-5) Website. https://www.ptsd.va.gov/professional/assessment/adult-sr/ptsd-checklist.asp. Accessed September 6, 2019.

Vonnahme, L. A., Lankau, E. W., Ao, T., Shetty, S., \& Cardozo, B. L. (2015). Factors associated with symptoms of depression among Bhutanese refugees in the United States. Journal of immigrant and minority health, 17(6), 1705-1714. 
Weathers, F. W., Litz, B. T., Keane, T. M., Palmieri, P. A., Marx, B. P., \& Schnurr, P. P. (2013). The ptsd checklist for dsm-5 (pcl-5). Scale available from the National Center for PTSD at www. ptsd. va. Gov.

Wood, G., Tyler, T. R., \& Papachristos, A. V. (2020). Procedural justice training reduces police use of force and complaints against officers. Proceedings of the National Academy of Sciences, 117(18), 9815-9821.

Yang, X., \& Mak, W. W. (2020). Addressing sociopolitical determinants of mental health: an emerging challenge in Hong Kong. The Lancet.

Xue, C., Ge, Y., Tang, B., Liu, Y., Kang, P., Wang, M., \& Zhang, L. (2015). A metaanalysis of risk factors for combat-related PTSD among military personnel and veterans. PloS one, 10(3), e0120270. 
Table 1.

Summary of sample characteristics per Depression and PTSD Diagnosis. These include age, sex, income level, number of protest attended, political extremism and ideology as well as exposure to police brutality, rubber ammunitions, teargas, experience of minor, severe injuries and custody ( $N$ $=522$ ).

\begin{tabular}{llcccc}
\hline \multirow{2}{*}{ Demographics } & & \multicolumn{2}{c}{ Depression } & \multicolumn{2}{c}{ PTSD } \\
\hline & & No $(\mathrm{n}=266)$ & Yes $(\mathrm{n}=256)$ & No $(\mathrm{n}=441)$ & Yes $(\mathrm{n}=81)$ \\
\cline { 2 - 5 } & Age $^{\dagger}$ & $45.1(12.9)$ & $42.9(12.2)$ & $44.4(12.6)$ & $41.7(12.5)$ \\
& $\%$ Male & 51.9 & 45.3 & 48.3 & 50.6 \\
& Income level $^{\ddagger}$ & $<30,000 €$ & $<30,000 €$ & $<30,000 €$ & $<30,000 €$
\end{tabular}

Activism

\begin{tabular}{|c|c|c|c|c|}
\hline Protests Attended ${ }^{\dagger}$ & $16.6(11.5)$ & 19.4(13.6) & $17.8(12.8)$ & 18.9(11.8) \\
\hline Extremism ${ }^{\dagger}$ & $1.36(1.15)$ & $1.29(1.15)$ & $1.34(1.16)$ & $1.21(1.14)$ \\
\hline Ideology ${ }^{\dagger}$ & $3.09(1.54)$ & $3.06(1.45)$ & $3.05(1.50)$ & $3.19(1.45)$ \\
\hline
\end{tabular}

Exposure

$\begin{array}{lcccc}\text { \% Police brutality } & 47.2 & 69.7 & 53.7 & 82.7 \\ \text { \% Rubber pellet } & 19.7 & 31.1 & 24.0 & 32.1 \\ \text { \% Teargas } & 81.2 & 90.6 & 83.4 & 98.8 \\ \text { \% Minor Injury } & 3.4 & 10.5 & 5.9 & 12.3 \\ \text { \% Severe injury } & 2.3 & 5.5 & 3.9 & 3.7 \\ \text { \% Custody } & 11.3 & 16.5 & 14.3 & 11.1\end{array}$

Note. Numbers for count variables (\%) represent frequencies. ${ }^{\dagger} \mathrm{Numbers}$ represent means and numbers between brackets SDs, ${ }^{\ddagger}$ Numbers represent median values. 
Table 2.

Summary of Correlation Analyses between HSCL, PCL scores, exposure to police brutality, rubber ammunitions and teargas, experience of minor, severe injuries and custody $(N=523)$.

\begin{tabular}{|c|c|c|c|c|c|c|c|c|}
\hline & 1 & 2 & 3 & 4 & 5 & 6 & 7 & 8 \\
\hline PCL Score & - & & & & & & & \\
\hline HSCL Score & $.84 * * *$ & - & & & & & & \\
\hline Police Brut. & $.29 * * *$ & $.33 * * *$ & - & & & & & \\
\hline Rubber Amm. & $.16 * * *$ & $.14 * *$ & $.29 * * *$ & - & & & & \\
\hline Teargas & $.21 * * *$ & $.23 * * *$ & $.38 * * *$ & $.23 * * *$ & - & & & \\
\hline Minor injury & $.19 * * *$ & $.20 * * *$ & $.17 * * *$ & $.16 * * *$ & $.11^{*}$ & - & & \\
\hline Severe injury & $.12 * *$ & $.12 * *$ & $.11 *$ & $.11 * *$ & .05 & $.46^{* * * *}$ & - & \\
\hline Custody & .07 & .07 & $.19 * * *$ & $.23 * * *$ & $.10 *$ & $.22 * * *$ & $.24 * * *$ & - \\
\hline
\end{tabular}




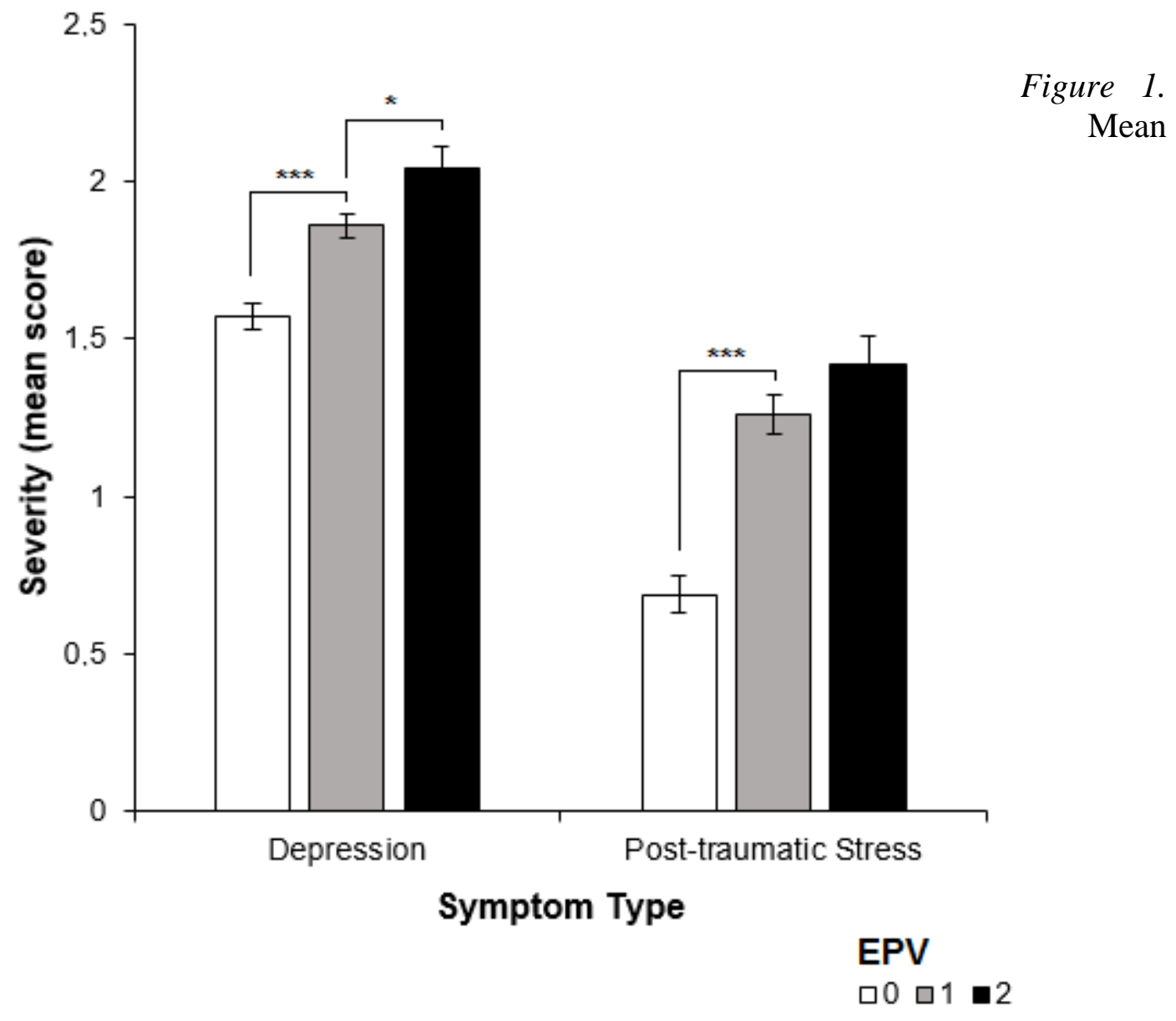

Depression and Post-traumatic Stress symptom severity scores across exposure groups. EPV $=$ Number of Exposures to Police Violence-related traumas. Error bars represent SE. $* * * p<.001, * p<.05$. 


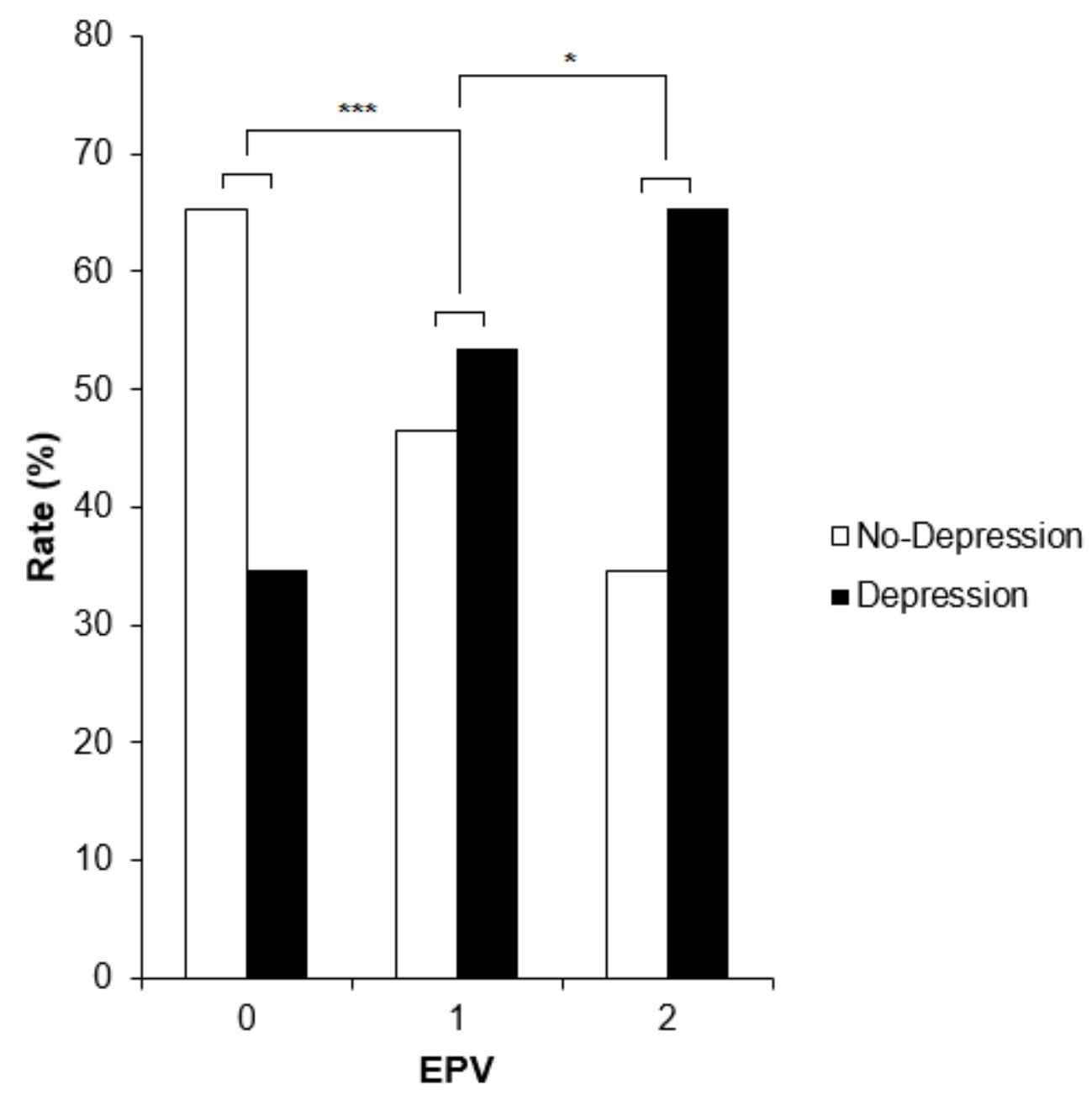

Figure 2. Tentative Depression rates across exposure groups. EPV = Number of Exposures to Police Violence-related traumas. $* * * p<.001, * p<.05$. 


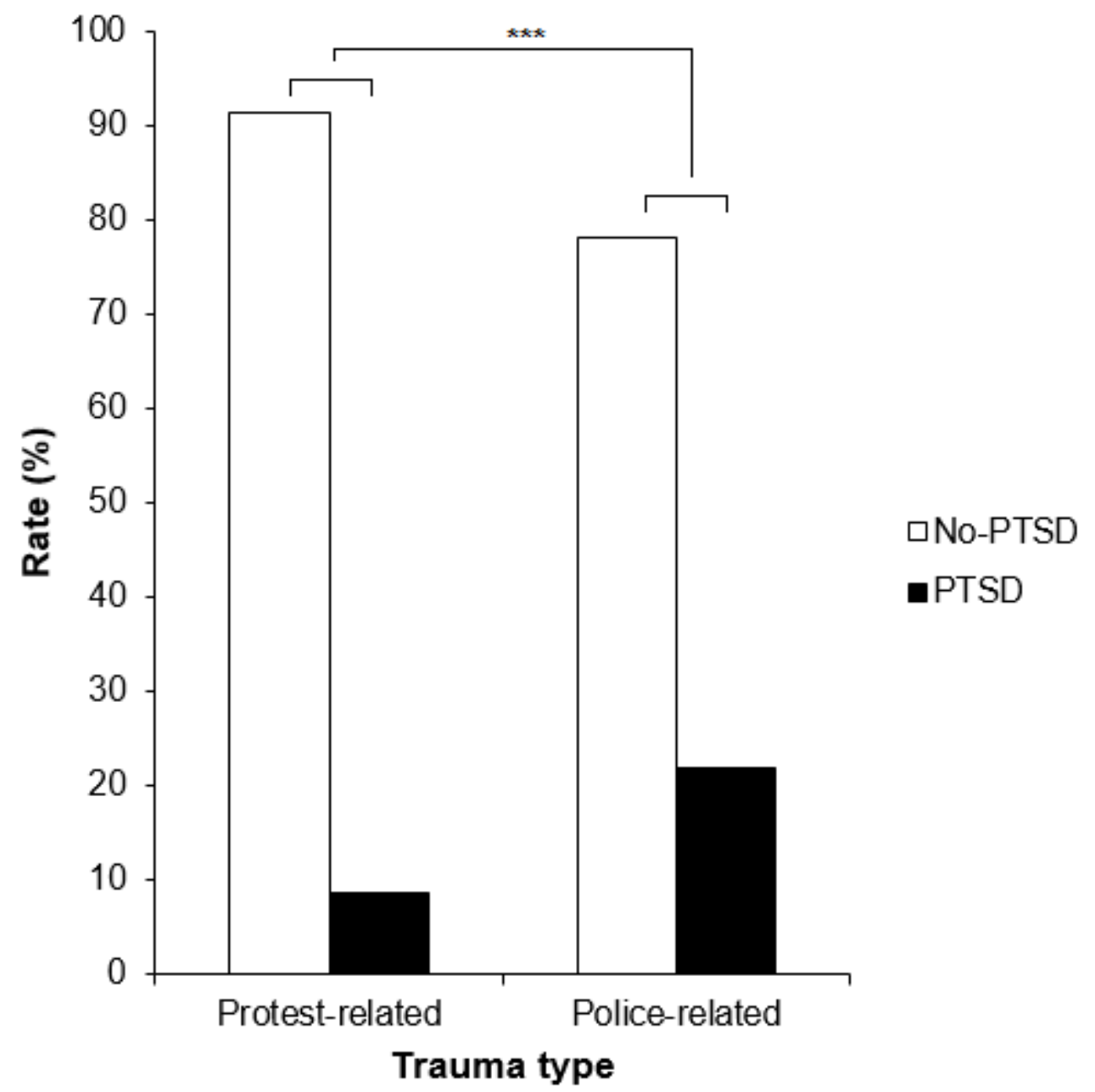

Figure 3. Tentative PTSD rates according to trauma type. $* * * p<.001$. 